Article

\title{
Preparation and Characterization of Carboxylic Acid Adducts of Gabapentin
}

\author{
Sunaree Choknud, Oratai Saisa-ard, and Kenneth J. Haller* \\ School of Chemistry, Institute of Science, Suranaree University of Technology, Nakhon Ratchasima 30000, \\ Thailand \\ E-mail: ken.haller@gmail.com*
}

\begin{abstract}
Gabapentin, $\mathrm{C}_{9} \mathrm{H}_{17} \mathrm{NO}_{2}, 2$-[1-(aminomethyl)cyclohexyl] acetic acid, is used as an anticonvulsant drug to help control partial seizures in the treatment of epilepsy and to manage postherpetic neuralgia after 'shingles'. Cocrystals of gabapentin with a series of hydroxyl carboxylic acids and a cocrystal of gabapentin with oxalic acid were previously reported. The adduct with salicylic acid was prepared, along with adducts of two dicarboxylic acids, succinic acid and adipic acid, and an adduct with 1,2,4,5benzenetetracarboxylic acid. Formation of new materials is demonstrated by new unique physical properties, including lower melting points $\left(102.8-105.1{ }^{\circ} \mathrm{C}, 90.0-93.0{ }^{\circ} \mathrm{C}\right.$, and 152.4-154.8 ${ }^{\circ} \mathrm{C}$ for succinic acid, adipic acid, and 1,2,4,5-benzenetetracarboxylic acid products, respectively) than those of the individual starting materials. Shifts in infrared bands for $v(\mathrm{O}-\mathrm{H}), v(\mathrm{~N}-\mathrm{H})$, and $v(\mathrm{C}=\mathrm{O})$ bands confirm adduct formation and indicate the nature of the interactions between the two components in the lattice.
\end{abstract}

Keywords: Cocrystal, gabapentin, 2-[1-(aminomethyl)cyclohexyl]acetic acid, adducts.

ENGINEERING JOURNAL Volume 16 Issue 3

Received 21 November 2011

Accepted 7 May 2012

Published 1 July 2012

Online at http://www.engj.org/

DOI:10.4186/ej.2012.16.3.29

This paper is based on the poster presentation at the German-Thai Symposium on Nanoscience and Nanotechnology 2011_Green Nanotechnology of the Future, GTSNN 2011, in Nakbon Ratchasima, Thailand, 13-16 September 2011. 


\section{Introduction}

Gabapentin, $\mathrm{C}_{9} \mathrm{H}_{17} \mathrm{NO}_{2}, 2$ 2-[1-(aminomethyl)cyclohexyl] acetic acid is a drug used as an anticonvulsant to help control partial seizures in the treatment of epilepsy. It reduces the number of seizures by increasing the amount of a closely related chemical called $\gamma$-aminobutyric acid (GABA) in the brain. It is also used in adults to manage a condition called postherpetic neuralgia, the pain that occurs after "shingles".

Pharmaceutical cocrystallization is of great interest in the field of drug development. Formation of cocrystals of pharmaceutical solids can often improve physicochemical properties, such as solubility, stability, bioavailability, hydroscopicity, and compaction behavior [1]. In the simplest sense cocrystals are crystalline molecular complexes containing two or more species with essentially molecular properties bound together in a single crystal lattice through noncovalent interactions [2-3]. Hydrogen bonds are the basis of molecular recognition phenomena in pharmaceutical systems and are responsible for the generation of families of periodic molecular networks with only one molecular component (single component crystals and their polymorphs) or with multiple molecular components (cocrystals or multiple component crystals, including solvates) in the crystalline state. The design of experiments to exploit formation of extended networks based on noncovalent interactions is a goal of crystal engineering.

Gabapentin contains strong hydrogen bond donor and strong hydrogen bond acceptor groups (see Fig. 1) which makes its participation in such networks possible. Four crystalline forms of gabapentin have been reported [4-6], attesting to its flexibility in forming supramolecular structures. Form I is gabapentin monohydrate, and Forms II, III, and IV are the $\alpha$-gabapentin, $\beta$-gabapentin, and $\gamma$-gabapentin polymorphs, respectively. Compounds containing carboxylic acid functional groups can readily interact with the $-\mathrm{NH}_{2}$ and $-\mathrm{COOH}$ fragments of un-ionized gabapentin, or the $-\mathrm{NH}_{3}{ }^{+}$and $-\mathrm{COO}^{-}$fragments of Zwitterionic gabapentin (Fig. 1) to form network synthons as seen in previous studies with GABA and gabapentin with carboxylic acids [7-10]. Melting points of the four known forms of gabapentin are given in Table 1.

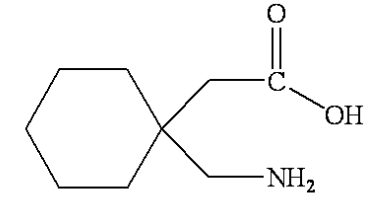

unionized gabapentin<smiles>O=C(O)CCCCC(=O)O</smiles><smiles>O=C(O)c1cc(C(=O)O)c(C(=O)O)cc1C(=O)O</smiles>

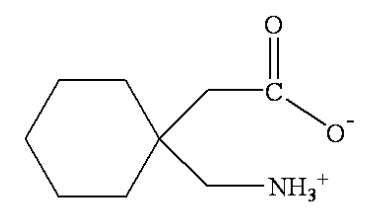

Zwitterionic gabapentin<smiles>O=C(O)CCC(=O)O</smiles><smiles>O=C(O)c1ccccc1O</smiles>

salicylic acid

benzene-1,2,4,5-tetracarboxylic acid

Fig. 1. Structural diagrams of gabapentin and the organic acids used in this study.

Cocrystallization of gabapentin with oxalic acid was reported in 2008 [9] and with a series of hydroxyl carboxylic acids in 2009 [10]. The 2008 study included a survey of the Cambridge Structural Database, CSD that identified over 12,000 instances of the particular bonding pattern as illustrated in Figure 2(a). The largest fraction of the CSD hits involves four individual moieties that are not covalently connected. Many involve two chemically different species, and thus, are cocrystalline or other multicomponent systems. In graph set notation this pattern is designated $\mathrm{R}_{4}{ }^{2}(8)$, where $\mathrm{R}$ identifies it as a ring, a cyclic pattern of bonds, 
the subscript denotes the number of hydrogen bond donors involved in the set, and the superscript denotes the number of hydrogen bond acceptors involved in the set [11]. An individual pattern of such supramolecular interactions is often called a synthon (or sometimes a supramolecular synthon), and can be understood as a logical extension of the concept of functional group originating in organic chemistry. While it is not necessary for a synthon to be symmetric, both synthons illustrated in Fig. 2 are often found in the solid state positioned about a crystallographic inversion center or exhibiting pseudo centrosymmety. The CSD survey finds 918 hits for structures exhibiting the $\mathrm{R}_{4}{ }^{2}(8)$ synthon in which the donor is an amino group $\left(-\mathrm{NH}_{2}\right)$ and the acceptor is a carbonyl group $(>\mathrm{C}=\mathrm{O})$. Ammonium donor $\left(-\mathrm{NH}_{3}{ }^{+}\right)$and carboxylate acceptor $\left(-\mathrm{COO}^{-}\right)$forms are also well represented in the CSD survey. The gabapentium oxalate report itself has an example of a crystallographically centrosymmetric $\mathrm{R}_{4}^{2}(8)$ synthon involving the ionized forms.

Table 1. Melting points of gabapentin polymorphs and gabapentin monohydrate.

\begin{tabular}{lccr}
\hline Form & Species & Melting point $\left({ }^{\circ} \mathbf{C}\right)$ & Reference \\
\hline I & gabapentin monohydrate & $156.0-156.7$ & {$[4]$} \\
II & $\alpha$-gabapentin & $160.0-162.0,155.0-166.0^{a}$ & {$[5-6]$} \\
III & $\beta$-gabapentin & $156.0-158.0,155.0-168.0^{a}$ & {$[5-6]$} \\
IV & $\gamma$-Gabapentin & $150.0-168.0^{a}$ & {$[6]$} \\
\hline
\end{tabular}

a Melting point measured by DSC [6].

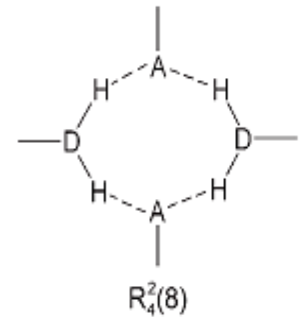

(a)

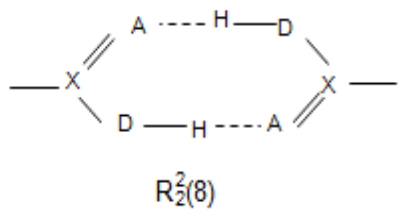

(b)

Fig. 2. Graph set notation for two simple synthons relating to carboxylic acids. a) an $\mathrm{R}_{4}{ }^{2}(8)$ synthon and b) the $\mathrm{R}_{2}{ }^{2}(8)$ synthon as found in a carboxylic acid dimer. D represents a hydrogen bond donor and $\mathrm{A}$ represents a hydrogen bond acceptor.

The present work reports preparation of adducts of gabapentin with two dicarboxylic acids, succinic acid and adipic acid, and an adduct with benzene-1,2,4,5-tetracarboxylic acid. Also, another adduct of gabapentin with salicylic acid was found while preparing the reported adduct from a modified solvent system. Formation of new materials is demonstrated by new unique physical properties, including melting points and morphologies. Shifts in infrared bands confirm adduct formation and indicate the nature of the interactions between the two components in the lattice.

\section{Experimental}

\subsection{Chemicals and Solvents}

Commercial chemicals: adipic acid (Acros Organics 99\%, laboratory grade), succinic acid (Carlo Erba 99.5\%, AR grade), benzene-1,2,4,5-tetracarboxylic acid, pyromellitic acid, (Acros Organics 96\%, laboratory grade), and salicylic acid (Mallinckrodt, AR grade), methanol (Carlo Erba 99.9\%, AR grade), and DI water.

Separation of gabapentin: Dissolve $7.49 \mathrm{~g}$, the content of 20 capsules, of Gabapentin drug (The Government Pharmaceutical Organization, Thailand, $300 \mathrm{mg}$ gabapentin/capsule) in $400 \mathrm{~mL}$ of methanol: $\mathrm{H}_{2} \mathrm{O}(3: 1 \mathrm{v} / \mathrm{v})$ 
and warm at $40{ }^{\circ} \mathrm{C}$ with stirring $(400 \mathrm{rpm})$ for $40 \mathrm{~min}$. Filter the hot solution and rinse the solids with cold DI water. Heat the filtrate at $50{ }^{\circ} \mathrm{C}$ until the volume is reduced to $130 \mathrm{~mL}$. Cool and let stand at room temperature for 3 days to crystallize. The colorless crystals were separated by vacuum filtration on a Buchner funnel and washed with $1 \mathrm{~mL}$ of cold DI water giving $5.10 \mathrm{~g}$ of gabapentin monohydrate $(77 \%$ recovery).

\subsection{Cocrystallization}

Gabapentin monohydrate $(378 \mathrm{mg}, 2 \mathrm{mmol})$ and adipic acid $(146 \mathrm{mg}, 1 \mathrm{mmol})$ were ground together 10 min, dissolved in $5 \mathrm{~mL}$ of methanol: $\mathrm{H}_{2} \mathrm{O}(2: 1 \mathrm{v} / \mathrm{v})$, and warmed at $37^{\circ} \mathrm{C} 20$ min with stirring, reducing the volume to $2.5 \mathrm{~mL}$. Crystalline product was obtained after 5 weeks of controlled evaporation at room temperature. Similar methodology was used for reactions of 2:1 mole ratio gabapentin with succinic acid, of 1:1 mole ratio gabapentin with benzene-1,2,4,5-tetracarboxylic acid, and of 1:1 mole ratio gabapentin with salicylic acid.

\subsection{Characterizations}

Crystal morphologies were observed with scanning electron microscopy (SEM) using a JSM 6400 electron microscope (JEOL, Japan). Infrared (IR) spectra were acquired on a Perkin-Elmer model spectrum GX Fourier transform infrared spectrophotometer in wave number range $400-4000 \mathrm{~cm}^{-1}$ from $\mathrm{KBr}$ pellets. Melting points were measured with a Stuart Model SMP 30 melting point apparatus (Bibby Scientific, UK).

\section{Results and Discussion}

Gabapentin was separated from commercially available drug capsules with high recovery using a methanol: $\mathrm{H}_{2} \mathrm{O}$ mixed-solvent system. Gabapentin crystals show rectangular crystal morphology as shown in Fig. 3. The product was characterized by melting point and IR spectroscopy. The measured melting point is $154.0-155.6^{\circ} \mathrm{C}$, which corresponds to the melting point of gabapentin monohydrate (Table 1, Form I) as well as to that of $\beta$-gabapentin (Form III).

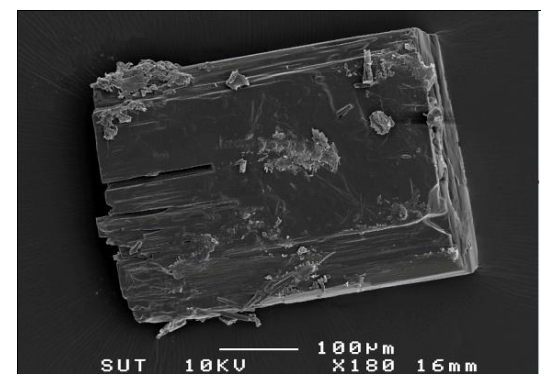

Fig. 3. SEM image of gabapentin crystal.

The infrared spectrum of the crystalline product is presented in Fig. 4. The $\mathrm{N}-\mathrm{H}$ stretching band, $\mathrm{v}(\mathrm{N}-$ $\mathrm{H})$, appears at $3286 \mathrm{~cm}^{-1}$ and the bending bands, $\delta_{\text {as }}(\mathrm{N}-\mathrm{H})$ and $\delta_{\mathrm{s}}(\mathrm{N}-\mathrm{H})$, at $1615 \mathrm{~cm}^{-1}$ and $1544 \mathrm{~cm}^{-1}$, respectively. A $\cup(\mathrm{N}-\mathrm{H})$ band at $2144 \mathrm{~cm}^{-1}$ corresponds to the additional vibrational band that often appears at about $2100 \mathrm{~cm}^{-1}$ when amine is converted to ammonium. Further, the $\mathrm{v}(\mathrm{C}=\mathrm{O})$ band is shifted lower at $1662 \mathrm{~cm}^{-1}$, compared to the typical $\mathrm{v}(\mathrm{C}=\mathrm{O})$ vibration of a carboxylic acid $\left(1700-1730 \mathrm{~cm}^{-1}\right)$, consistent with the partial delocalization that often occurs in a carboxylate ion. The $\mathrm{v}(\mathrm{C}-\mathrm{O})$ band of carboxylate shows at $1400 \mathrm{~cm}^{-1}$. The band at $3389 \mathrm{~cm}^{-1}$ is assigned to $\mathrm{v}(\mathrm{O}-\mathrm{H})$ of water, suggesting the product is gabapentin monohydrate. The total spectrum is quite similar to gabapentin form I reported in the previous work [12], confirming the product to be gabapentin monohydrate. 


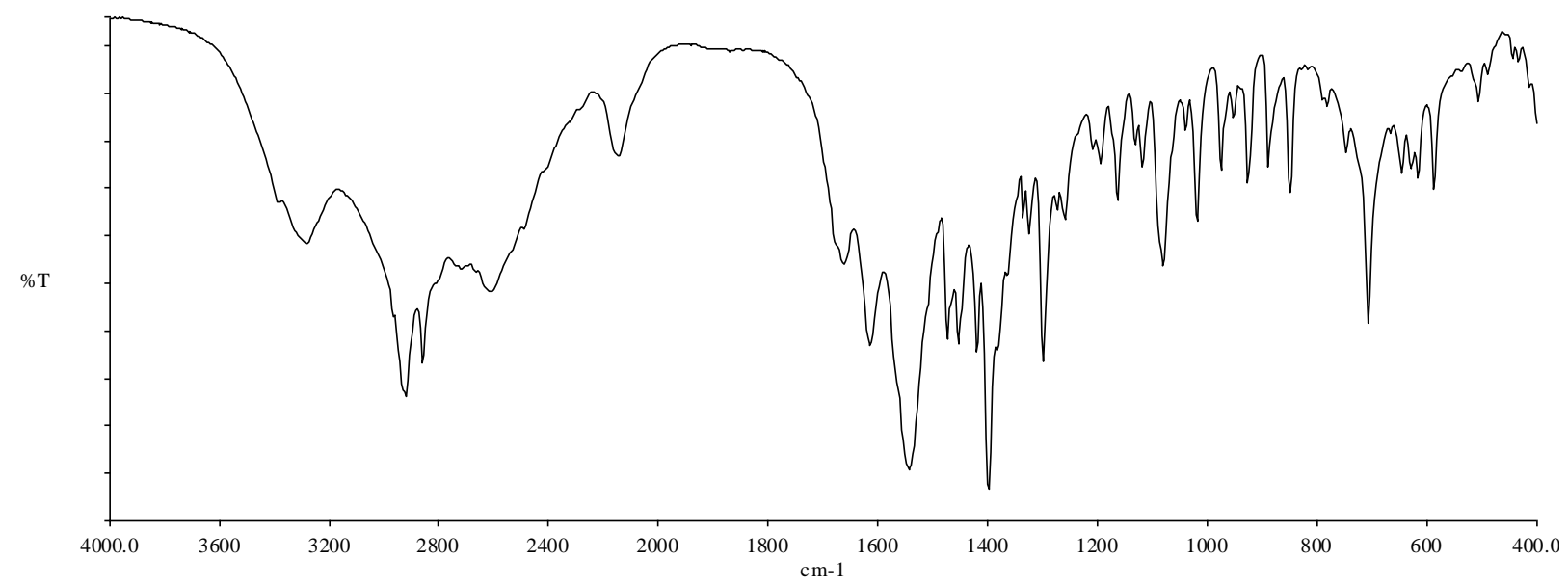

Fig. 4. IR spectrum of gabapentin monohydrate.

Morphologies of the solid products of the cocrystallization experiments, observed with SEM, are shown in Fig. 5. SEM images of adducts of gabapentin with adipic acid, succinic acid, and benzene-1,2,4,5tetracarboxylic acid (Fig. 5(a-c)) show plate morphology while the crystalline product for the adduct of gabapentin with salicylic acid shows prism morphology.
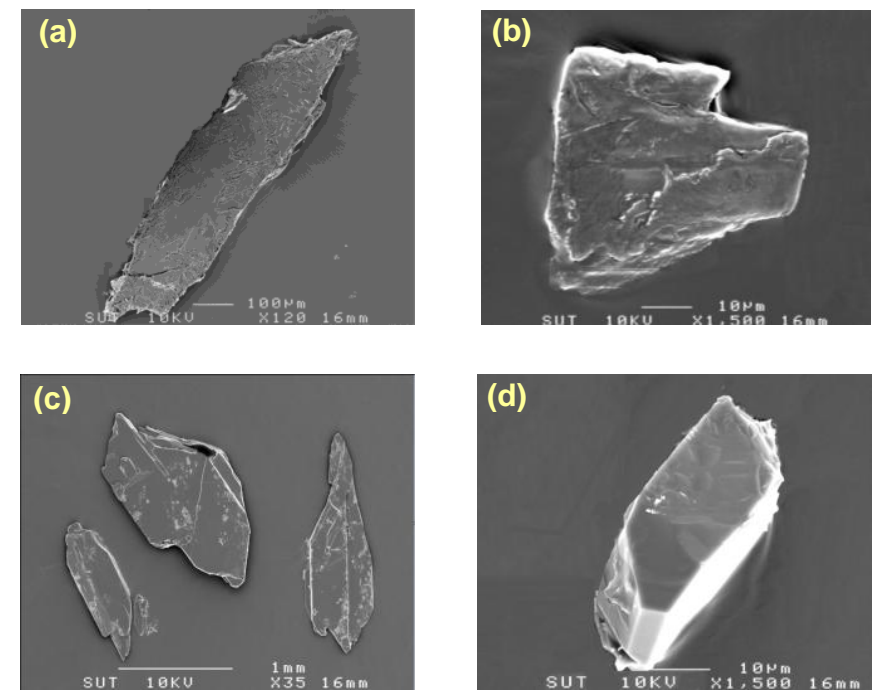

Fig. 5. SEM images of adducts of gabapentin with (a) adipic acid, (b) succinic acid, (c) benzene-1,2,4,5tetracarboxylic acid, and (d) salicylic acid.

Formation of new materials is demonstrated by new unique physical properties, including lower melting points than the starting materials (Table 2). The melting points of the solid product adducts of gabapentin with succinic acid and with salicylic acid are quite similar and very much reduced, similar to the adduct with malic acid [10]. The melting point of the solid product from reaction of gabapentin with salicylic acid is lower (about $30^{\circ} \mathrm{C}$ ) than the $131{ }^{\circ} \mathrm{C}$ previously reported [10]. The narrow range of the current melting point determination suggests it is indeed a pure compound, and thus is a new adduct of gabapentin with salicylic acid.

Infrared spectra of the adducts were compared to the spectra of both gabapentin and the relevant carboxylic acid starting materials to determine the identity of the solids isolated from the cocrystallization experiments. The band positions and assignments for the solid products are summarized in Table 3 .

The IR result for the gabapentin:salicylic acid adduct is consistent with the previous report [10], but shows new peaks at $1670-1690 \mathrm{~cm}^{-1}$ and $1633 \mathrm{~cm}^{-1}$ when compared to the previous work. These two peaks are assigned to $\mathrm{v}(\mathrm{C}=\mathrm{O})$ of carboxylate group and $\delta(\mathrm{N}-\mathrm{H})$ of ammonium ion, respectively. 
Table 2. Melting points of reactants and multicomponent crystalline products.

\begin{tabular}{ccc}
\hline & Material & Melting point $\left({ }^{\circ} \mathbf{C}\right)$ \\
\hline reactants & gabapentin & $154.0-155.6$ \\
adipic acid & 152.0 \\
succinic acid & 185.0 \\
& salicylic acid & 281.0 \\
& benzene-1,2,4,5-tetracarboxylic acid & 159.0 \\
adducts & gabapentin:adipic acid & $90.0-93.0$ \\
& gabapentin:succinic acid & $102.8-105.1$ \\
& gabapentin:benzene-1,2,4,5-tetracarboxylic acid & $152.4-154.8$ \\
gabapentin:salicylic acid & $102.0-105.0$ \\
\hline
\end{tabular}

Gabapentin:adipic acid adduct: The IR spectrum contains peaks due to both reactant species. Peaks at 2930 and $2859 \mathrm{~cm}^{-1}$ are assigned to the $\mathrm{v}(\mathrm{C}-\mathrm{H})$ of gabapentin. The $\mathrm{v}(\mathrm{N}-\mathrm{H})$ band of gabapentin is shifted to lower energy $\left(3229 \mathrm{~cm}^{-1}, \Delta \mathrm{v}_{(\mathrm{N}-\mathrm{H})}=57 \mathrm{~cm}^{-1}\right)$ indicating the $-\mathrm{NH}_{3}+\cdots \mathrm{O}^{-}$interaction of the ionized ammonium of gabapentin with a carboxylate group. The $\mathrm{v}(\mathrm{C}=\mathrm{O})$ of a carboxylic acid group at $1702 \mathrm{~cm}^{-1}$ is shifted to higher energy compared to both reactants, indicating an $-\mathrm{O}-\mathrm{H}^{\cdots} \mathrm{O}^{-}$interaction of the carboxylic acid group of gabapentin with a carboxylate of adipic acid with the possiblity that an $\mathrm{R}_{4}^{2}(8)$ hydrogen bond synthon has formed. The band at $1645 \mathrm{~cm}^{-1}$ corresponds to $\mathrm{v}(\mathrm{C}=\mathrm{O})$ of carboxylate groups. While similar discussion of 2:1 gabapentin:oxalic acid adducts has been reported [9], the possibility of formation does not prove the synthon has formed as is illustrated by the GABA:oxalic acid study $[7,8]$ which contains the ionized ammonium and carboxylate forms, but does not contain the $\mathrm{R}_{4}{ }^{2}(8)$ hydrogen bond synthon.

Gabapentin:succinic acid adduct. The IR spectrum shows peaks at 2935 and $2859 \mathrm{~cm}^{-1}$ due to $\mathrm{v}(\mathrm{C}-\mathrm{H})$ and peaks at 1714 and $1644 \mathrm{~cm}^{-1}$ attributed to $\mathrm{v}(\mathrm{C}=\mathrm{O})$ of carboxylic acid and carboxylate, respectively. The $\mathrm{v}(\mathrm{N}-\mathrm{H})$ band was observed as a weak broad band at $3258 \mathrm{~cm}^{-1}$, shifted $51 \mathrm{~cm}^{-1}$ to lower energy when compared to the gabapentin spectrum, indicating an $-\mathrm{NH}_{3}+\cdots \mathrm{O}^{-}$interaction of the ionized ammonium of gabapentin with a carboxylate of succinic acid. The $\mathrm{v}(\mathrm{C}=\mathrm{O})$ peak shifted to higher energy at $1725 \mathrm{~cm}^{-1}$ when compared to the succinic acid spectrum, similar to $\mathrm{v}(\mathrm{C}=\mathrm{O})$ in the gabapentin:adipic acid adduct suggesting similar formation of hydrogen bond synthons in the structures.

Gabapentin:benzene-1,2,4,5-tetracarboxylic acid adduct: The $\mathrm{v}(\mathrm{O}-\mathrm{H})$ bands at 3532 and $3389 \mathrm{~cm}^{-1}$ are observed in the product spectrum with no peak shift compared to the benzene-1,2,4,5-tetracarboxylic acid spectrum, suggesting the carboxylic acid groups do not change to the carboxylate form. There are two $\mathrm{v}(\mathrm{C}=\mathrm{O})$ bands at $1690-1712 \mathrm{~cm}^{-1}$ region indicating different types of hydrogen bond formation between the carboxylic acid groups of benzene-1,2,4,5-tetracarboxylic acid with Zwitterionic gabapentin. Vibrational bands at 1590 and $1626 \mathrm{~cm}^{-1}$ are assigned to $\delta(\mathrm{N}-\mathrm{H})$ of the ionic $-\mathrm{NH}_{3}{ }^{+}$and $\mathrm{v}(\mathrm{C}=\mathrm{O})$ of carboxylate, respectively. Similar discussion of 1:1 gabapentin:trimesic acid adducts has been previously reported [13].

Gabapentin:salicylic acid adduct: The $\mathrm{v}(\mathrm{N}-\mathrm{H})$ band at $3253 \mathrm{~cm}^{-1}$ is shifted $33 \mathrm{~cm}^{-1}$ to lower energy compared to free gabapentin. A very broad peak in the $3300-2700 \mathrm{~cm}^{-1}$ region indicates hydrogen bond interactions between the $-\mathrm{NH}_{3}{ }^{+}$group of gabapentin and a $-\mathrm{COO}^{-}$carboxylate group. Two $\mathrm{v}(\mathrm{C}=\mathrm{O})$ peaks at 1677 and $1713 \mathrm{~cm}^{-1}$ shifted to higher frequencies compared to the reactants indicates that the carbonyl group is involved in hydrogen bond interaction(s). A previous report on a 1:1 adduct of gabapentin and salicylic acid [10] shows intramolecular interaction between $-\mathrm{O}-\mathrm{H}$ and a carboxylate group in salicylic acid, and intermolecular $-\mathrm{NH}_{3}+\cdots \mathrm{O}^{-}$interactions between ionized ammonium of gabapentin with the carboxylate group of ionized salicylic acid. The solid product from the previous work shows a significantly higher melting point than the product of this work. The current product also shows additional peaks at 1670-1690 $\mathrm{cm}^{-1}$ and $1633 \mathrm{~cm}^{-1}$ in the IR spectrum. These two peaks are assigned to $\mathrm{v}(\mathrm{C}=\mathrm{O})$ of carboxylate group and 
$\delta(\mathrm{N}-\mathrm{H})$ of ionized ammonium, respectively. These results indicate the formation of a new gabapentin:salicylic acid adduct.

Table 3. IR spectral peak positions and assignment of gabapentin monohydrate and solid product peaks.

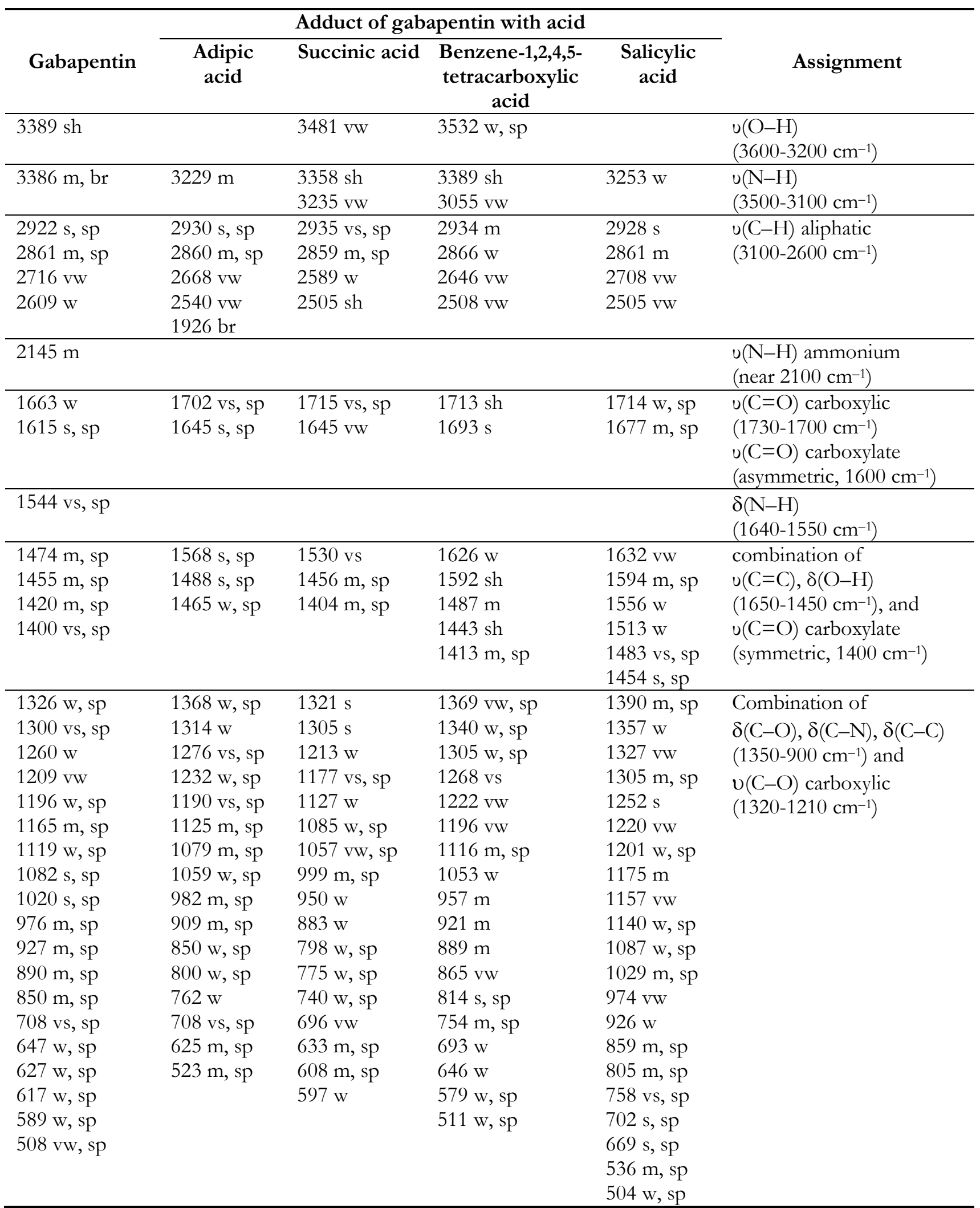

Abbreviations: $v s=$ very strong, $s=$ strong, $m=$ medium, $w=$ weak, $v w=$ very weak, $s h=$ shoulder, $s p=$ sharp, $b r=$ broad; $v=$ stretching and $\delta=$ bending. 


\section{Conclusion}

Pure gabapentin was separated from the drug Neurontin with high recovery, $85 \%$ based on the quantity of gabapentin in a capsule. Three new solid state molecular complexes; 2:1 gabapentin:adipic acid, 2:1 gabapentin:succinic acid, and gabapentin:benzene-1,2,4,5-tetracarboxylic acid adduct were formed. The product combining gabapentin with salicylic acid shows a lower melting point than the adduct reported in published work. Shifts of infrared bands and lower melting points compared to the individual starting materials indicate adduct formation and the nature of the interactions between the two components in the lattice.

\section{Acknowledgements}

We thank SUT Cooperative Program for arranging Sunaree Choknud's undergraduate research project at Suranaree University of Technology during her undergraduate study (in the Department of Chemistry, Faculty of Science, Ubon Ratchathani Ratjabhat University, Ubon Ratchathani) and Suranaree University of Technology for laboratory and instrumentation support. Oratai Saisa-ard thanks the Thai Commission Higher Education (CHE) for a Ph.D. scholarship.

\section{References}

[1] B. Sekhon, "Pharmaceutical co-crystals-a review," ARS Pharm., vol. 50, pp. 99-117, 2009.

[2] A. Jayasankar, A. Somwangthanaroj, Z. J. Shao, and N. Rodríguez-Hornedo, "Cocrystal formation during cogrinding and storage is mediated by amorphous phase," Pharm. Res., vol. 23, pp. 2381-2392, 2006.

[3] A. V. Trask, "An overview of pharmaceutical cocrystals as intellectual property," Mol. Pharm., vol. 4, pp. 301-309, 2007.

[4] D. E. Butler and B. J. Greenman, US patent 4894476, 1990.

[5] S. Kalyan and A. Pradesh, "Process for the preparation of gabapentin," U.S. Patent 0187295, Aug. 25, 2005.

[6] H. A. Reece and D. C. Levendis, "Polymorphs of gabapentin," Acta Cryst., vol. C64, pp. o105-o108, 2008.

[7] M. Wenger and J. Bernstein, "Designing a cocrystal of $\gamma$-amino butyric acid," Angew. Chem. Int. Ed. (Engl.), vol. 45, pp. 7966-7969, 2006.

[8] N. Khosavithitkul and K. J. Haller, "Supramolecular structure of cocrystallized $\gamma$-amino butyric acid and oxalic acid," Chiang Mai J. Sci., vol. 38, pp. 405-411, 2011.

[9] M. Wenger and J. Bernstein, "An alternate crystal form of gabapentin: a cocrystal with oxalic acid," Cryst. Growth Des., vol. 8, pp. 1595-1598, 2008.

[10] L. S. Reddy, S. J. Bethune, J. W. Kampf, and N. Rodríguez-Hornedo, "Cocrystals and salts of gabapentin: $\mathrm{pH}$ dependent cocrystal stability and solubility," Cryst. Growth Des., vol. 8, pp. 378-385, 2009.

[11] J. Bernstein, R. E. Davis, L. Shimoni, and N.-L. Chang, "Patterns in hydrogen bonding: functionality and graph set analysis in crystals," Angew. Chem., Int. Ed. (Engl.), vol. 34, pp. 1555-1573, 1995.

[12] C.-H. Hsu, W.-T. Ke, and S.-Y. Lin, "Progressive steps of polymorphic transformation of gabapentin polymorphs studied by hot-stage FTIR microspectroscopy," J. Pharm. Pharmaceut. Sci., vol. 13, pp. 6777, 2010.

[13] V. André, A. Fernandes, P. P. Santos, and M. T. Duarte, "On the track of new multicomponent gabapentin crystal forms: synthon competition and pH stability," Cryst. Growth Des., vol. 11, pp. 2325 2334, 2011. 